\title{
Simulation and experimental verification of dose distributions of electron beams
}

\author{
J. ŠEMNICKÁ ${ }^{1,2}$, J. KLUSOŇ ${ }^{1}$
}

(Manuscript received 29 June 2011, accepted 2 November 2011)

ABSTRACT Accurate dose calculations are very important in radiotherapy treatment planning. The Monte Carlo method has proven to be an accurate and reliable method for simulation of the dose distributions from electron beams and can provide theoretically more accurate distributions than conventional planning system algorithms (e.g. the Generalized Gaussian Pencil Beam algorithm) for more complex target configurations. The aim of this work was to assess the parameters and spectrum of a given electron beam (Varian CLINAC 2100 C/D) from experimental depth dose distribution and then simulate dose distributions in an experimental arrangement with a 3D polymer gel dosimeter. The comparison of data from experimental measurements, Monte Carlo calculations and the planning system enabled us to verify experimentally the simulation model and technique as well as the planning system results. Both simple and more complex systems (e.g. dose distributions close to different material interfaces) can be studied. The generation and influence of the "bremsstrahlung" photons in the electron beams was also analyzed using the simulation technique. The computations, experimental methods used and results obtained are presented and discussed.

Keywords: Monte Carlo simulation / electron beams / 3D gel dosimetry / treatment planning systems

RÉSUMÉ Simulation et vérification expérimentale des distributions de dose des faisceaux d'électrons

Les calculs précis sont très importants dans la planification des traitements en radiothérapie. La méthode Monte Carlo est éprouvée comme exacte et fiable pour la simulation des distributions de dose des faisceaux d'électrons. Elle peut fournir théoriquement les distributions plus exactes que les algorithmes des systèmes conventionnels de planification (par ex., l'algorithme Generalized Gaussian Pencil Beam) pour des configurations à cible plus complexe. Ce travail a eu pour but d'évaluer les paramètres et le spectre du faisceau d'électrons (Varian CLINIC $2100 \mathrm{C} / \mathrm{D}$ ) de la distribution expérimentale de la dose profonde et ensuite de simuler les distributions de dose dans un arrangement expérimental au moyen d'un dosimètre en gel de polymères $3 \mathrm{D}$. La comparaison des données mesurées expérimentalement avec les calculs Monte Carlo et avec le système de planification permet de vérifier expérimentalement le modèle de simulation et sa technique ainsi que les résultats du système de planification. Des systèmes simples ou complexes peuvent être étudiés (par ex., les distributions de dose autour des interfaces des matériaux différents). La production et l'influence des photons de « bremsstrahlung » dans les faisceaux d'électrons ont été aussi étudiées à l'aide de la simulation. Les méthodes de calcul et les techniques expérimentales utilisées sont présentées et les résultats discutés.

Czech Technical University in Prague, Faculty of Nuclear Sciences and Physical Engineering, Brehova 7, Prague 1, 115 19, Czech Republic.

2 Na Homolce Hospital, Medical Physics Department, Roentgenova 37/2, Prague 5, 150 00, Czech Republic. 


\section{Introduction}

Electron radiotherapy is preferably used for superficially located lesions and is indicated in approximately $5-10 \%$ cases. The treatment plan usually consists of one field defined by a suitable electron cut-out inserted in an applicator. The patient dose distribution is calculated and visualized in a treatment planning system (TPS) using patient CT images. Modern TPSs provide dose calculation algorithms based on a pencil beam approach or Monte Carlo method (MC); the pencil beam approach is still widely clinically used. Electron pencil beam algorithms exploit the Fermi-Eyges theory, (e.g., Jette et al., 1988), which describes broadening of a pencil beam due to multiple scattering along its path. In general, the implementation of pencil beam algorithms is realized in the following steps:

(1) The beam area around the calculation point is divided into discrete elements.

(2) A pencil beam is ray-traced through each element. Heterogeneities are taken into account using either semi-infinite slab approximation (i.e. various tissues are modeled as if they were a stack of slabs, infinite in lateral extent) or more sophisticated three-dimensional algorithms (Hyödynmaa, 1991).

(3) The Fermi-Eyges theory is applied to obtain the width of each pencil beam at the depth of dose calculation. The dose is thus the sum of contributions from all pencil beams.

Since the Fermi-Eyges theory does not include depth absorption of the beam it is necessary to renormalize the calculated dose to a measured depth dose curve for reference geometry. Another drawback of the Fermi-Eyges theory is that it does not describe large-angle single-scattering events, which do not obey the Gaussian model. This can result in errors in profiles and calculation of output factors.

In the Eclipse (Varian, USA) TPS, on which this work was focused, the Generalized Gaussian Pencil Beam (GGPB) algorithm incorporates threedimensional heterogeneity correction published by Hyödynmaa (1991) and improved modeling of radial dose profiles by including large-angle scatter via summing a few Gaussian functions (Lax et al., 1983). Despite these improvements, users can observe "hot and cold" spots close to surfaces in between regions of more scattered pencils and less scattered pencils due to the varying degree of lateral charged particle equilibrium (Ahnesjö, 2010).

The goal of this work was to experimentally verify the relative dose distribution calculated by the Eclipse GGPB using a gel dosimetry phantom and the MC method. The following section describes details of the gel dosimetry measurement and the Monte Carlo model. 


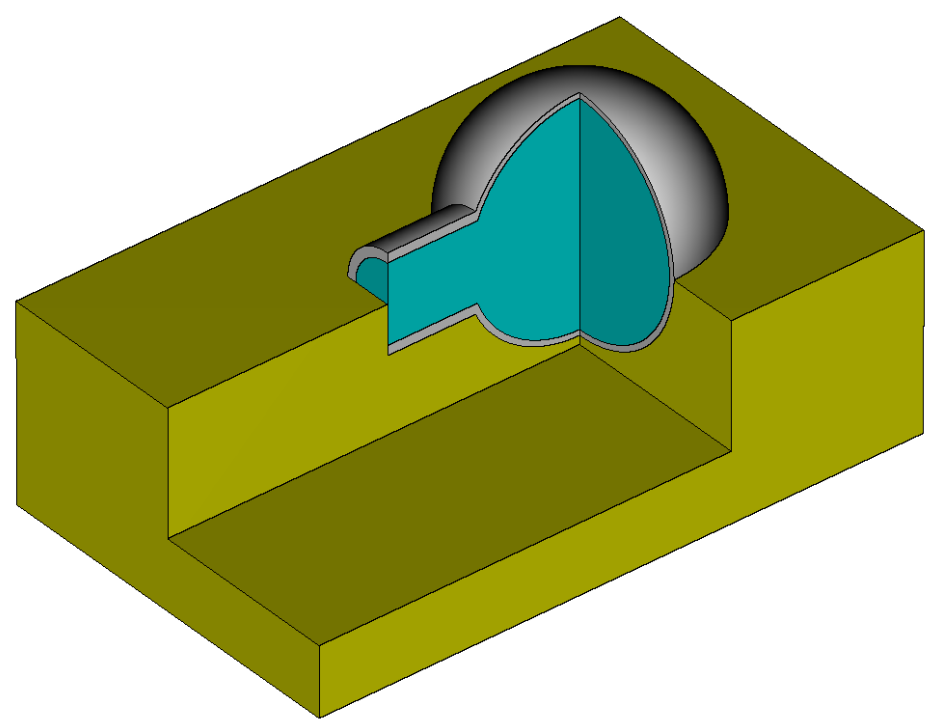

Figure $1-3 D$ view of the MCNPX gel phantom model.

\section{Materials and methods}

\subsection{Treatment planning system}

The version of the Eclipse TPS in this experiment was 6.5, build 7.3.10.

\subsection{Gel dosimetry phantom and Eclipse calculation}

The gel phantom consisted of a spherical glass flask $(250 \mathrm{ml})$ filled with a polymer gel complemented with a cork stand (see model in Fig. 1 for illustration). A polyacrylamide gel was used (5\% gelatine, $3 \% \mathrm{~N}, \mathrm{~N}$ '-methylene-bisacrylamide, $3 \%$ acrylamide), with linear response up to ten grays (Novotny et al., 2002). The phantom was first filled with water and scanned on CT ( $2 \mathrm{~mm}$ slice), and a single $9 \mathrm{MeV}$ field plan was generated in Eclipse using the GGPB algorithm (the calculation grid was $1.25 \mathrm{~mm}$; the size of the pencil beam was $2.5 \mathrm{~mm}$ ). The source to surface distance (SSD) was set to $100 \mathrm{~cm}$ to the surface of the glass flask. The field size was $10 \times 10 \mathrm{~cm}$ defined by a cerrobend cut-out. The maximum prescribed dose was $7 \mathrm{~Gy}$ and the gel was irradiated 2 days after its manufacture.

The gel dosimeter response was read out using clinical magnetic resonance scanners (Magnetom Expert 1T and Trio 3T, both Siemens). The 1T scanner 
provided a response in the central slice of the phantom while the $3 \mathrm{~T}$ provided a full set of 3-dimensional data. Scanning sequences and their parameters are summarized in Table I. It was assumed that the gel dose-R2 response is linear and, therefore, calibration tubes were not needed.

\subsection{Monte Carlo simulation}

Description of the model geometry and all calculations were made using the MCNPX 2.5.0 computer code (Pelowitz at al., 2005).

\section{(1) Geometry}

The model of the gel phantom (Fig. 1) was described according to the real experimental arrangement and materials as realistically as possible including gel composition. Lattice type 1 was used to voxelize the gel active volume according to the CT and planning system calculation grid.

\section{(2) Source}

Very simple geometry of the beam with a point isotropic electron source placed in a vacuum at a distance of $100 \mathrm{~cm}$ above the flask surface was used. The square field was defined by a mathematical plane with zero importance to cut out the shape of the square field. The plane was placed $5 \mathrm{~cm}$ above the flask surface.

To obtain the energy spectrum, a method published by Wei et al. (2007) was used. The method exploits a set of monoenergetic percent depth dose curves (PDDs) whose linear combination should yield the measured central axis PDD.

TABLE I

Parameters of the scanning sequences.

\begin{tabular}{ccc}
\hline Parameter & Trio 3T & Expert 1T \\
\hline Sequence type & Fast spin echo (3D) & Multiple spin echo (2D) \\
\hline Time from irrad. to scanning & 17 days & 60 hours \\
TE [ms] & 12,809 & 22.5 (16 echoes) \\
TR [ms] & 14421 & 2000 \\
\hline Slice thickness [mm] & 2 & 2 \\
\hline Number of averages & 3 & 9 \\
\hline Pixel size [mm] & $0.94 \times 0.94$ & $1 \times 1$ \\
Flip angle [deg] & 120 & 180 \\
Pixel bandwidth [Hz] & 130 & 1 \\
\hline Echo Train length & 65 & $50 \% / 50 \%$ \\
FoV phase & $100 \% / 100 \%$ & $256 / 256$ \\
FoV read & $128 / 128$ & \\
\hline
\end{tabular}


The coefficients therefore represent the energy spectrum. We simulated monoenergetic depth dose distributions from $375 \mathrm{keV}$ up to $10750 \mathrm{keV}$ with $125 \mathrm{keV}$ increment in the same beam geometry, but instead of the gel phantom we defined a large water phantom of $\varnothing 50 \times 50 \mathrm{~cm}$. The deposited energy in water per one history was scored on a field central axis in small $4 \times 4 \times 1 \mathrm{~mm}$ voxels.

Contamination of the electron beam by the bremsstrahlung photons originating in the treatment head and collimation system was not included in the model of the source.

(3) Tallies

Tally *F8 and selected lattice planes were used for calculation of dose distributions. Spherical mesh tally type 3 was set to study the dose distribution on the glass-water interface more precisely. Rectangular mesh tallies type 1 for scoring of electron and photon energy deposition were set (correspondingly with the lattice) to analyze secondary photon contributions and individual tally characteristics.

(4) Settings

The ITS energy indexing algorithm was used in electron transport simulation. Considering the small voxel size the ESTEP parameter was set to 20 as a compromise between recommendations for substep length and computing time. Relative error in voxels in the region of maximum of PDD $\left(\mathrm{PDD}_{100}\right)$ is less than $1 \%$, for $\mathrm{PDD}_{50}$ it is about $1.8 \%$, about $3 \%$ for $\mathrm{PDD}_{10}$ and less than $5 \%$ for $\mathrm{PDD}_{5}$.

\subsection{Data processing}

All data were manipulated and compared in Matlab 6.5 (The Mathworks, USA).

The Eclipse dose distribution for the contoured flask volume was exported in DICOM format. The grid size was kept the same as the calculation grid. It was necessary to cut out the volume corresponding just to the inner gel volume (no glass wall).

Dose maps (R2 maps) from gel measurements were calculated from T2 weighted images using the software of each particular MR scanner. Before dose (R2) map normalization, background corresponding to $0 \mathrm{~Gy}$ was subtracted. The background value was obtained from a non-irradiated flask.

From the results of the MC calculation, only *F8 energy tallies were used for quantitative comparison. The results of the spherical mesh tally were used only for qualitative or visual assessment. The results of the rectangular mesh tally type 1 were compared with the *F8 tally. 


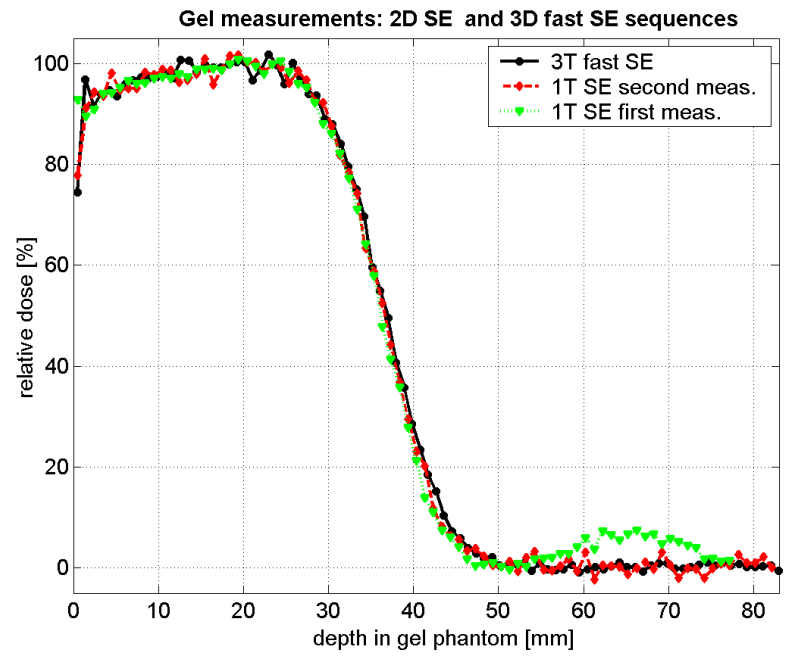

Figure 2 - Results of all gel measurements, central axis PDD.

In order to perform comparisons, it was necessary to normalize all data sets in the same way. As the normalization point the maximum value on the central percent depth dose curve (PDD) was used. The maximum was determined by fitting experimental, simulated and Eclipse PDDs by polynomial functions.

To compare the two-dimensional dose distributions, gamma analysis (Low et al., 1998) was used with $4 \%$ dose difference (DD) and $3 \mathrm{~mm}$ distance in agreement with (DTA) criteria, and furthermore with $3 \%$ DD and $2 \mathrm{~mm}$ DTA criteria to reveal subtle differences in compared data. The gamma index is a useful index using combined criteria (dose and distance) to assess agreement of two data sets and is especially useful for data where steep dose gradients occur. If the index exceeds unity it means that the compared data sets differ more than selected criteria in a particular pixel (or point).

\section{Results}

\subsection{Performance of the gel dosimeter}

The central PDDs obtained from gel measurements are depicted in Figure 2. The figure includes a third curve, which represents the second independent measurement with a different gel batch. It is obvious that all data are in agreement within the region of the steepest dose gradient. In the first gel measurement in the 


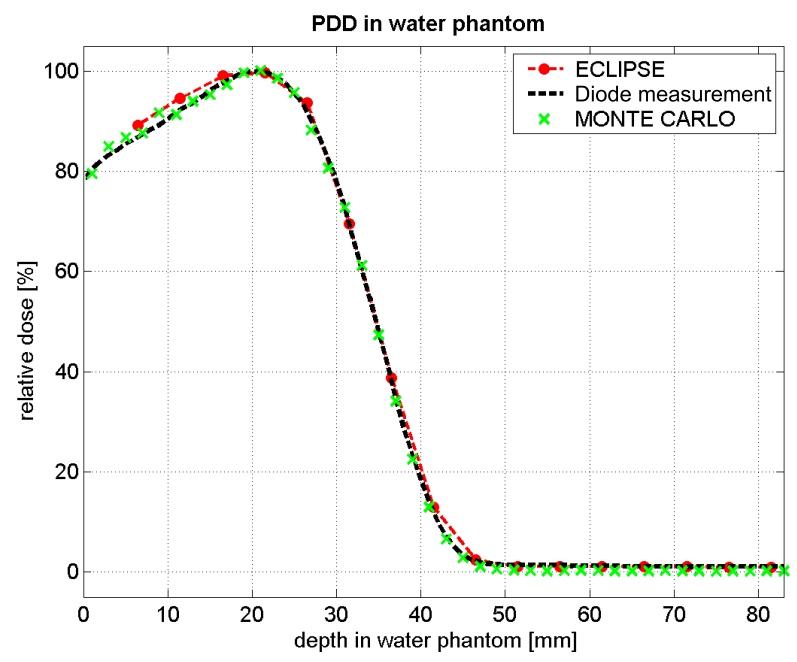

Figure 3 - Evaluation of the $9 M e V$ beam MC model-large water phantom PDDs.

low dose region a local maximum can be seen. This rise was explained as a MR artifact as it was not observed in the measurement at the 3T scanner. Also, for this reason the gel experiment was repeated including another gel manufacture.

\subsection{Monte Carlo model}

The electron energy spectrum plays a crucial role in the designed model. It was therefore important to evaluate the model for an elementary geometry. With the unfolded spectrum a simulation for the large water phantom was performed and compared with the Eclipse calculation in a large water phantom. Figure 3 shows the Eclipse calculation, MC simulation (tally *F8) and diode measurement. All these central PDDs agree as expected.

\subsection{Comparison of measured, simulated and planned dose distributions}

Figure 4 shows the central PDDs of the Eclipse calculation, gel measurement and MC simulation. The corresponding central transversal two-dimensional dose distributions are displayed in Figure 5.

Figures 6 and 7 show the results of gamma analysis for $4 \%, 3 \mathrm{~mm}$ and $3 \%$, $2 \mathrm{~mm}$, respectively. From these figures one can see that the gel measurement differs from the Eclipse calculation in the region of the steep dose gradient. The MC tally *F8 lies virtually between the gel measurements and Eclipse calculation 


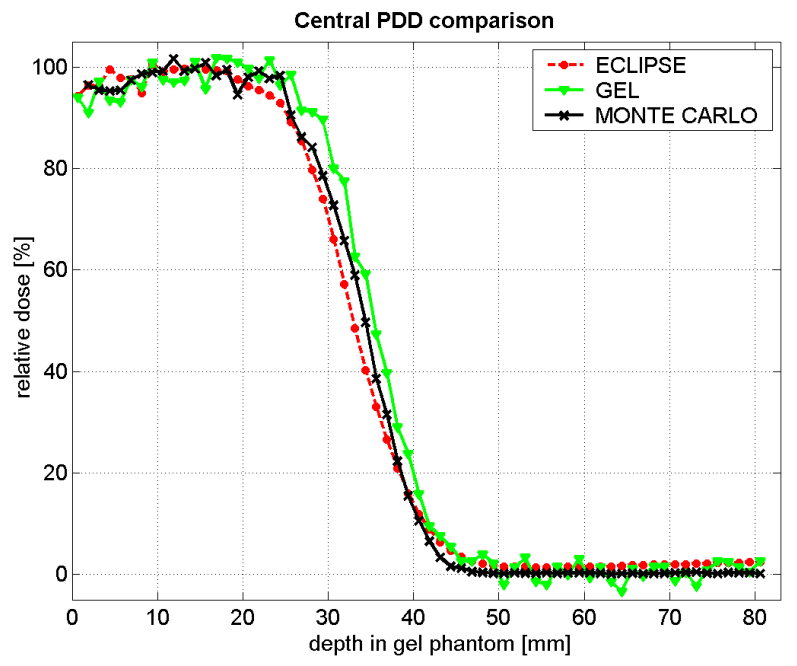

Figure 4 - Central axis PDD from Eclipse, gel measurement and MC.

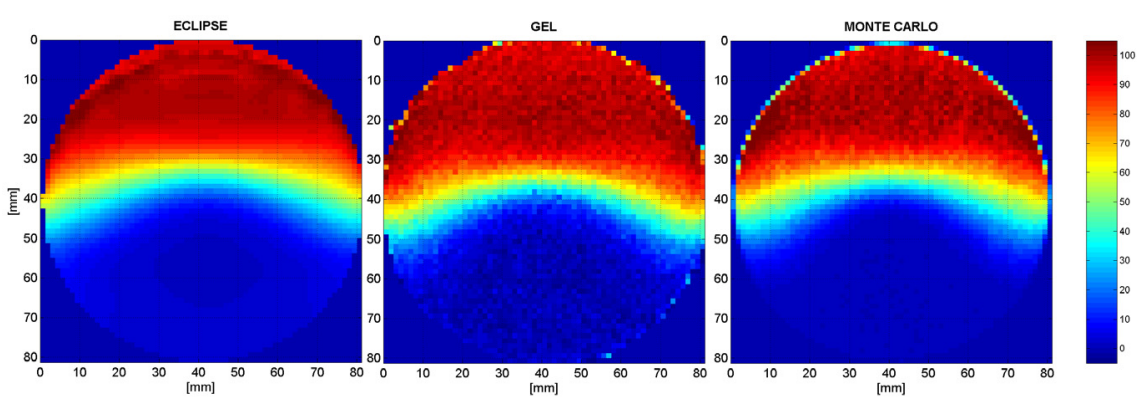

Figure 5 - Two-dimensional dose distributions.

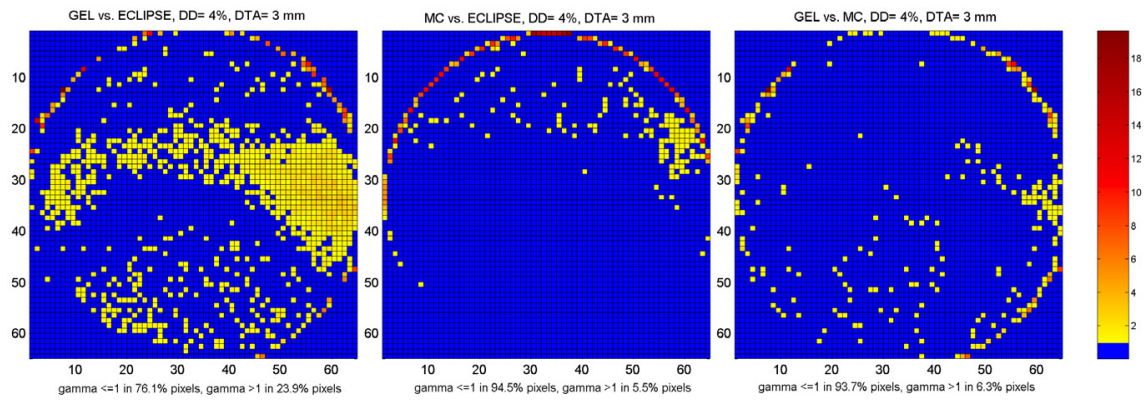

Figure 6 - Results of gamma analysis, $4 \% \mathrm{DD}, 3 \mathrm{~mm}$; the scale is now expressed in pixels (1 pixel =1.25 mm). 


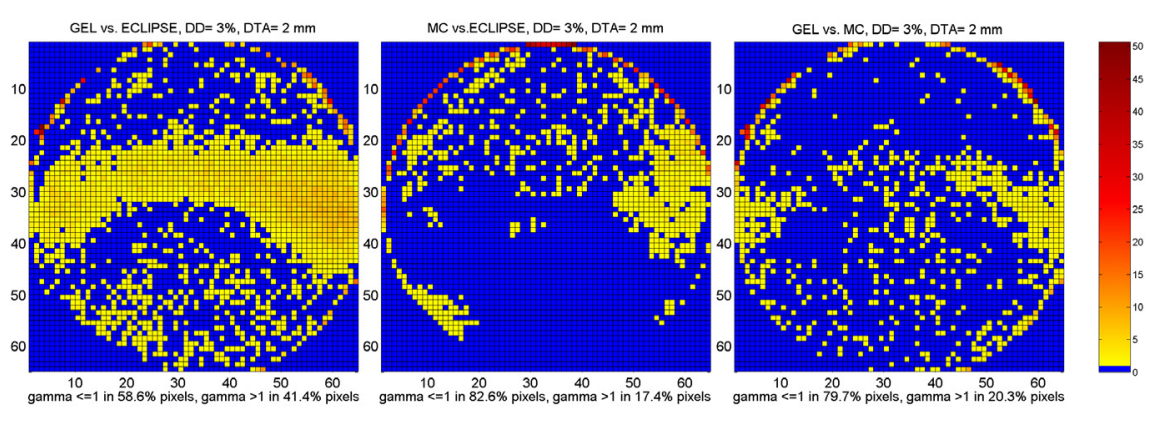

Figure 7 - Results of gamma analysis, $3 \% \mathrm{DD}, 2 \mathrm{~mm}$; the scale is now expressed in pixels (1 pixel =1.25 mm).

and does not confirm the accuracy of the gel measurements. The same is shown in the two-dimensional maps of the gamma index (blue represent values less than or equal to one). Higher values of the gamma index are also present close to the edges of the spherical flask. This cannot be considered as discrepancy since the way the flask contour was defined differed for each modality. In the Eclipse data, the glass shell had to be cropped in order to compare the data with the simulation and the gel measurement. In gel measurements the gel contour was affected by noise and was difficult to define. The MC gel phantom was defined as an ideal sphere, and, therefore, it differs from the real shape of the glass container.

The spherical mesh tally is depicted in Figure 8. The values represent deposited energy per volume (not dose), but still, it allows inspecting the energy deposition close to the surface of the glass flask and gel filling. It can be concluded that no "hot and cold" spots are present and this feature of the GGPB algorithm can be considered as a calculation artifact.

The results for the rectangular mesh tally type 1 (pedep option) were expected to provide an estimation of the dose contribution from phantom-generated "bremsstrahlung" photons. Figure 9 shows the central PDD obtained from the *F8 tally, and the electron and photon dose PDD obtained from the mesh tally type 1 results. The results of the mesh tally 1 were expressed as absorbed energy per volume, but the values of the total dose inside the flask were normalized using exactly the same procedure as for the data obtained from the *F8 tally. The maximum value of the phantom bremsstrahlung reaches about $0.4 \%$ of the maximum electron dose. Relative errors of the calculated dose in the region beyond the electron range (which is important for reconstruction of headgenerated bremsstrahlung contribution from experimental data) are about $30-40 \%$ for the $* \mathrm{~F} 8$ and collision heating tallies and about $6-7 \%$ for the track-length heating photon dose estimation, so that further improvements of the model and simulation technique will be necessary. 


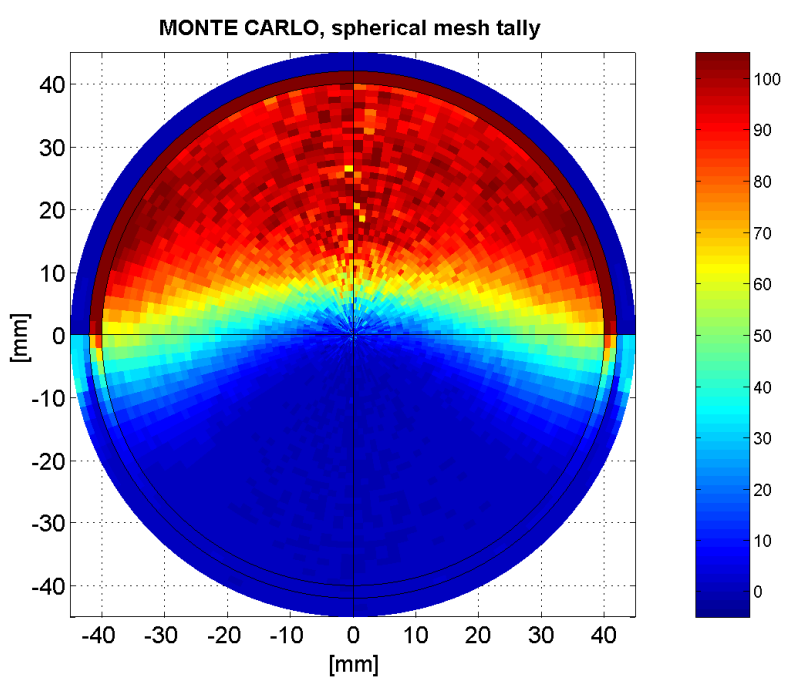

Figure 8 - Spherical mesh tally, energy per volume, normalized to the maximum on the central axis.

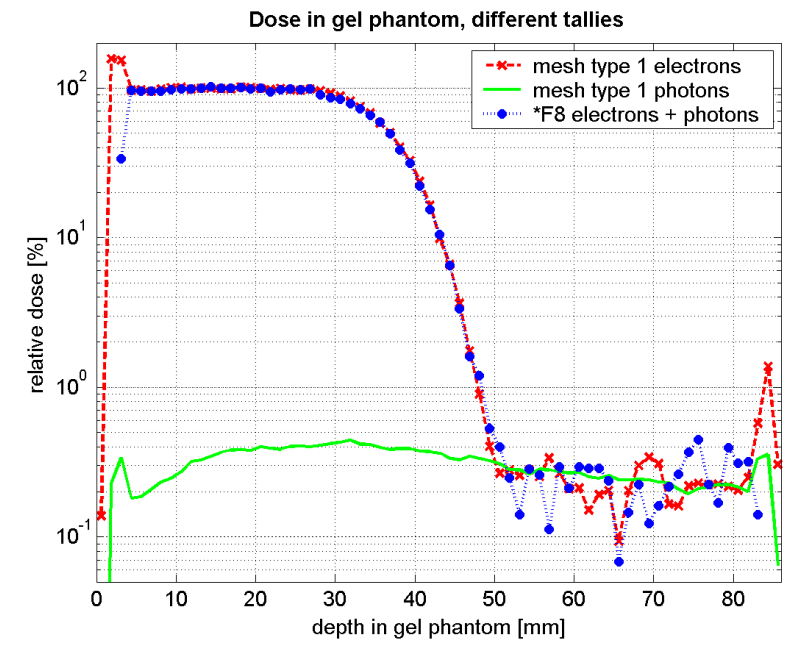

Figure 9 -Comparison of the *F8 tally with the mesh tally type 1 for electrons and photons - central axis $P D D$ in the gel phantom. The maxima in shallow depth on mesh type curves (red and green) represent absorbed energy per $\mathrm{cm}^{-3}$ in the glass flask. 


\section{Discussion}

\subsection{Gel measurements and Eclipse calculation}

A gel dosimeter is an integral dosimeter with no or minimal LET or dose rate dependence (Novotny et al., 2001). The performed measurements show good reproducibility and, most probably, reflect the real situation. The discrepancy between the gel and Eclipse results can probably be explained by the failure of the TPS algorithm for this phantom configuration, but more study will be necessary. On the other hand, it is only fair to remark that the phantom does not reflect a real clinical case, and in reality such curved surfaces (small spheres) are not present in patients, or, at least, are encompassed by water-equivalent boluses. Probably a cylindrical phantom would be a better choice.

\subsection{Monte Carlo model}

Considering the simplicity of the designed beam model, the agreement between the gel measured and calculated data is good. The beam model did not include real beam angular distribution. Angular distribution is influenced by collimator scatter producing large-angle scattered electrons. This feature is also not perfectly modeled in the TPS algorithm. Most likely this is the reason why the regions of the largest deviations between the gel measurements and MC calculation appear on the sides of the gel phantom (Fig. 7, on the right).

The model does not include bremsstrahlung photons generated in the collimation system and the linac head. The head-generated bremsstrahlung contribution for a $9 \mathrm{MeV}$ beam may reach $1.6 \%$ of the maximum electron dose, depending on the linac head configuration (Zhu et al., 2001). Within the procedure for spectral reconstruction the bremsstrahlung tail was subtracted using extrapolation of the measured data beyond the electron practical range. This is in fact a simplification and as the spectral reconstruction is a badly conditioned problem, it might lead to errors in the reconstructed spectrum. However, the input data, i.e., the monoenergetic PDDs, for the reconstruction were MC-generated and therefore noisy, which can also be translated into spectral errors.

Further improvements of the beam model can be achieved with proper handling of the head-generated "bremsstrahlung" contribution in the spectral reconstruction procedure as well as in generating input data of higher quality (i.e., monoenergetic PDDs). Methods for separation of the electron and photon dose experimentally are described in (Zhu et al., 2001). From the measurements of the PDDs it is possible to determine the ratio of photon dose relative to the electron dose. The photon PDD 
can be used to reconstruct the energy spectrum of the head-generated photons in a similar fashion as was done for the electron energy spectrum.

\subsection{MC tallies}

Different types of tallies were tested and compared from the point of view of result interpretation, errors and runtime requirements. No substantial differences in runtime for lattice and mesh tallies were found. For calculation of dose distributions from bremsstrahlung, especially in the region beyond the electron range, the track-length heating tally for photons is most effective.

\section{Conclusion}

It was demonstrated that modern electron treatment planning algorithms might not generate accurate results for complex phantom (or patient) configuration, which demonstrates the need for their independent verification. In this work two different methods were used. One was purely experimental, 3D gel dosimetry, and the other, the $\mathrm{MC}$ method, combined experimental input data with computational procedures.

The gel dosimetry results demonstrate that if the gel dosimetry protocol is well established, gel dosimetry is an ideal 3D dosimeter/phantom. Further investigations and development in the field of gel dosimetry and its use in medical applications are ongoing and are planned for the future.

A simplified MC beam model was designed. Despite its simplicity the results obtained showed good agreement with the gel measurements and the TPS calculation. The model can be theoretically generalized for other field sizes and nominal beam energies. Further improvements can be achieved by modeling of the bremsstrahlung photons originating in the linac treatment head.

Acknowledgment. This research was supported by the Ministry of Education, Youth and Sports of the Czech Republic under project no. SGS10/090/OHK4/1T/14 and project no. MSM6840770020, and by The Czech Science Foundation under project No. 202/09/H086.

\section{REFERENCES}

Ahnesjö A. (2010) Beam characterization and dose modelling for electron beams, ESTRO Teaching Course on Dose Modelling and Verification for External Beam Radiotherapy, Seville, Spain, 14-18 March 2010.

Hyödynmaa S. (1991) Implementations of the generalized Gaussian pencil beam algorithm for threedimensional electron beam dose planning, Espoo: Technical Research Centre of Finland. ISBN 951-38-3943-5. 
Jette D. et al. (1988) Electron dose calculation using multiple-scattering theory. A: Gaussian multiplescattering theory. Med. Phys. 15 (2), 123-137.

Lax I. et al. (1983) Electron beam dose planning using Gaussian beams. Improved radial dose profiles, Acta Radiol. Suppl. 364, 49-59.

Low D.A. et al. (1998) A technique for the quantitative evaluation of dose distributions, Med. Phys. 25 (5), 656-661.

Novotny J. et al. (2001) Energy and dose rate dependence of BANG-2 polymer-gel dosimeter, Med. Phys. 28 (8), 2379-2387.

Novotny Jr. J. et al. (2002) Quality control of the stereotactic radiosurgery procedure with the polymergel dosimetry, Radiotherapy and Oncology, 63 (6), 223-230.

Pelowitz D.B. et al. (2005) MCNPX ${ }^{\mathrm{TM}}$ 2.5.0, Monte Carlo N-Particle Transport Code System for Multiparticle and High Energy Applications, LA-CP-05-0369 (2005).

Wei J. et al. (2007) Reconstruction of electron spectra from depth doses with adaptive regularization, Med. Phys. 33 (2), 354-359.

Zhu T.C. et al. (2001) Characteristics of bremsstrahlung in electron beams, Med. Phys. 28 (7), 1352-1358. 